\title{
Numerical Investigations of Polyester Coextrusion Instabilities
}

\author{
O. Mahdaoui ${ }^{a}$ and P. Laure ${ }^{b, a}$ and J.-F. Agassant ${ }^{a}$ \\ ${ }^{a}$ Mines ParisTech, CEMEF, UMR CNRS 7635, Sophia Antipolis, 06560, Valbonne, \\ France \\ ${ }^{b}$ Laboratoire J. A. Dieudonné, UMR CNRS 7351, Université de Nice, Parc Valrose, \\ 06108 Nice Cedex 02, France
}

\begin{abstract}
Interfacial instabilities occurring in the coextrusion process of molten polymers have been widely studied. The theoretical work based on the stability of 2D Poiseuille multilayer flows (invariant along the flow motion) have pointed out the convective nature of this instability (it is either amplified or damped in the flow direction). This behaviour has been confirmed by experimental observations. As this instability is purely elastic (the Reynold number in coextrusion process is very small), it is necessary to introduce a viscoelastic constitutive equation for the polymers. Comparisons between experimental observations and theoretical approaches based on the Giesekus or White-Metzner models give good agreements for laboratory simple devices. However, the applicability of this approach for industrial coextrusion process remains an important challenge, because one has to deal with the influence of complex geometries, varying temperature and more realistic constitutive equations. As it is still very costly in computational time to perform 3D instationary computations for viscoelastic fluids, a methodology to analyse the appearance of interfacial instabilities in a complex geometry is proposed within this paper. First, a stationary $3 \mathrm{D}$ computation is performed in the real geometry for a single polymer. This computation allows to find zones in which the flow motion is essentially $2 \mathrm{D}$, isotherm and with maximum values of the shear rate and, thus, the Weissenberg number. Instationary 2D finite element computations for two layer flows and a multi-mode differential viscoelastic model are then performed in these zones. Spatio-temporal analyses give information on the spatial behaviour of interfacial disturbances. This methodology is applied to the coextrusion of two polyesters for which
\end{abstract}


experimental observations are available. The simulations predict well the experimental results and show that the outlet zone can amplify low frequencies perturbations. Finally, the influence of processing parameters (temperatures, flow rates, ...) and die geometries has been checked.

Keywords: Coextrusion, interface instability, rheology, Spatio-temporal analysis

\section{Introduction}

The coextrusion process consists in extruding different polymers in the same die in order to get a multi-layer product which combines the properties (mechanical, optical, adhesion, barrier) of the different layers. It allows for example to substitute lacquer for steel protection in food packaging industry. Here, the coextruded film is composed of two layers, the bottom one providing good adhesion properties on the steel sheet substrate, and the upper one good printing property. The steel coated sheet will then be deep-drawn to shape for example a beverage can. The two polymer layers are constituted of various polyester patented formulations which present very different rheological properties. For some processing conditions, coextrusion instabilities are observed between the two layers. They induce unacceptable optical properties for the final steel coated product (figure 1).

Coextrusion instabilities have been experimentally investigated for a long time. Three kinds of experimental studies have been performed.

- In the first approach, the transition between stable and unstable processing conditions is investigated by examining the aspect of the extrudate obtained with different couples of commercial polymers (Han and coworkers [1, 2, 3], Antukar et al. [4], Valette et al. [5]). The authors related stable and unstable processing conditions to thickness ratio as well as viscosity and first normal stress difference ratios at the interface.

- In the second approach, the coextrusion flow and the development of instabilities have been examined in a very long die between parallel plates: after stopping the rotation velocity of the two extruders, the die is quickly cooled down, then dismounted and the interface between the two incompatible polymers is carefully analysed [6]. The instability 
is not visible at die entrance, but then develops and amplifies along the die length, pointing out its convective character.

- In the third approach, the instability is studied by looking at its development inside a partially transparent die for slightly unstable processing conditions, that is to say near the transition between stable and unstable coextrusion conditions. In Khomami and coworkers papers $[7,8,9,10,11]$ evolution of the interface deviation along the die is observed through four optical windows for various wave numbers of the interface defect and various layer thickness ratios (or flow rate ratios). In Valette et al. paper [12] the same phenomenon has been observed, but the lateral walls of the channel die are now totally transparent, which allows a precise analysis of the progressive development of the instability. Interface perturbations are generated by adding small amplitude periodic oscillations of the flow rate at die entrance. These perturbations grow or decay as they travel downstream, pointing clearly out the convective nature of the interfacial instability [13].

From a theoretical point of view, the occurrence of such instabilities has been largely studied by means of a linear stability analysis of the two-layer Couette or Poiseuille flow. In such analysis, one studies the growth or decay rate of periodic and arbitrary small perturbations using the Gasters transformation [14]. In the twenty past years, stability analysis have been carried out for different viscoelastic constitutive equations (see for example $[15,16,17,18])$. All these studies point out that instability occurs even at very low Reynolds number and is mainly governed by the viscosity ratio, the elasticity ratio and the thickness ratio of the two layers of polymers.

More recently, authors use more realistic constitutive equations to compare linear stability analysis results with experiments. Ganpule and Khomami $[19,20]$ use a 4-mode Giesekus constitutive equation, as Valette et al. [6] use a White-Metzner constitutive equation. There is a quantitative agreement with experimental observations both in terms of critical flow rate ratio and spatial amplification of interfacial disturbances.

For the first time Valette et al. [13] computed numerically the behaviour in time and space of an interfacial perturbation generated at the inlet of a Poiseuille flow of two coextruded Maxwell fluids of high viscosity. The perturbation moves as a wave packet in the flow direction with a velocity different from that of the interface and is spatially damped or amplified according to parameter values (viscosity, elasticity and flow rate ratios). Due 
to the high viscosity of both polymers, the Reynolds number is close to zero and the driving parameter of the instability is the Weissenberg number which balances the first normal stress difference and the shear stress. As usual, theses previous non dimensionless numbers are defined thanks to characteristic length and velocity of apparatus by taking

$$
R e=\frac{\rho U_{\mathrm{ref}} L_{\mathrm{ref}}}{\eta} \quad ; W e=\lambda \frac{U_{\mathrm{ref}}}{L_{\mathrm{ref}}},
$$

where $\eta, \rho$ and $\lambda$ are respectively the viscosity, the density and the relaxation time of fluid. For more complex fluids for which the relaxation time and viscosity are shear dependant, new expressions of theses non dimensionless numbers are given at the end of the next section. This numerical analysis of the effect of a small perturbation was then compared to the linear stability analysis of the two-layer Poiseuille flow and a good agreement was found.

Time-dependent viscoelastic computations involve two major difficulties $[21,22]$. First, the non-linearity and the hybrid type of constitutive equations (elliptic and hyperbolic) make the convergence of the iterative algorithm difficult especially with geometrical or boundary conditions singularities. The second difficulty is the time-dependent computation of the interface. For these reasons, time dependent viscoelastic computations of multi-layer flow have been performed with rather simple rheological models, at moderate Weissenberg numbers and in simple geometries [13, 23, 24]. The competition between surface tension force and elastic stress for mono-layer flows is also explored in $[25,26]$.

The aim of this paper is to perform a numerical study of the propagation of an interface perturbation in real die geometries and with realistic constitutive equations for the molten polymers. First we use a 3D finite element computation of the whole coat-hanger die geometry (with only one polymer). The velocity is maximal at the die inlet and at the die exit and it is supposed that instabilities will originate in these flow regions where the Weissenberg number is a maximum. In addition the flow pattern is $2 \mathrm{D}$ in these two flow regions. So we develop a $2 \mathrm{D}$ multilayer viscoelastic model with a precise capturing method of the interface. Then we apply this method to the inlet zone and the exit zone geometry of the coat-hanger die for various initial instability frequencies and for various processing conditions where defects are (or are not) experimentally observed. Finally we test some modifications of the geometry and of the processing conditions. 


\section{Presentation of coextrusion process and polymers}

We consider a geometry composed of three zones (figure 2): the inlet zone is a feeding channel with a narrow width and an important thickness; then there is a distribution channel with a complex geometry (called coat hanger geometry) which allows to distribute regularly the polymer flow through the die width $(350 \mathrm{~mm})$. The third broad zone is of uniform thin thickness (1 $m m)$.

The two coextruded polymers (PET1 and PET2) are polyester based patented formulations. They present very different rheological properties which have been measured using a cone and plate RMS 800 rheometer. Dynamic rheology measurements give the complex modulus (usually called $G^{\prime}$ and $\left.G^{\prime \prime}\right)$ for various temperatures. By assuming the Cox-Merz rule, viscosity

$\eta$ (the norm $\sqrt{\left(G^{\prime} / \omega\right)^{2}+\left(G^{\prime \prime} / \omega\right)^{2}}$ of complex viscosity)) and relaxation time $\left(\lambda=G^{\prime} / \omega G^{\prime \prime}\right)$ are deduced. The bottom layer (PET1) in contact with the steel sheet has good adhesive properties and marked viscoelastic behaviour. The upper layer (PET2) is classical polyester with a quasi-Newtonian behaviour. Figure 3 shows the master curve for the viscosity of both polymers at $260^{\circ} \mathrm{C}$ and figure 4 the master curve for the relaxation time at the same temperature. The activation energy of both polymers is very different: $E=38 \mathrm{~kJ} /$ mole for PET1 and $E=61 \mathrm{~kJ} /$ mole for PET2.

This makes this coextrusion situation particularly interesting. At the reference temperature $\left(260^{\circ} \mathrm{C}\right) \mathrm{PET} 1$ is much more viscous than PET2 within the extrusion processing range (typically 10 to $100 \mathrm{~s}^{-1}$ ) but both viscosities become equivalent at higher rates. When decreasing the temperature the viscosity of PET2 increases much more than the viscosity of PET1 and so the gap between viscosities of both polymers decreases and the shear rate for which the viscosities are equivalent decreases towards the range of processing conditions. Following the literature $([1,2,3,4])$ one may believe a strong influence of temperature and flow rate on the defect appearance. Figure 4 shows that the mean relaxation time is much more important for PET1 than for PET2 in the extrusion processing range.

As a consequence PET2 may be considered at a first glance as a Newtonian fluid ( $=582$ Pa.s at the reference temperature $260^{\circ} \mathrm{C}$ ).

PET1 behaviour is fitted with two constitutive equations:

- A Carreau-Yasuda equation for the viscosity will be used for the 3D 
computation:

$$
\eta(\dot{\bar{\gamma}}, T)=\eta_{0} \exp \left[\frac{E}{R}\left(\frac{1}{T}-\frac{1}{T_{0}}\right)\right]\left[1+(\lambda \dot{\bar{\gamma}})^{a}\right]^{\frac{m-1}{a}}
$$

where $\eta_{0}$ is the Newtonian plateau viscosity at the reference temperature $T_{0}\left(\eta_{0}=4791\right.$ Pa.s at $\left.260^{\circ} \mathrm{C}\right), \dot{\bar{\gamma}}$ is the second invariant of the rate of strain tensor,

$$
\dot{\bar{\gamma}}=\sqrt{2 \sum_{i, j} \dot{\epsilon}_{i, j}^{2}}
$$

$R$ is the universal gas constant, $\lambda(0.34), a(0.42)$ and $m(0.48)$ are fitting parameters.

- A multimode Johnson-Segalman model [27] will be used for the 2D time dependent computations:

$$
\begin{gathered}
\sigma=-p \mathbb{I}+2 \eta_{s} \dot{\epsilon}(u)+\tau ; \tau=\sum_{i} \tau_{i} \\
\tau_{i}+\lambda_{i}\left(\left(1-\frac{b}{2}\right) \frac{\delta \tau_{i}}{\delta t}+\frac{b}{2} \frac{D \tau_{i}}{D t}\right)=2 \eta_{i} \dot{\epsilon}(u)
\end{gathered}
$$

$\eta_{s}$ is a solvent viscosity $\left(\eta_{s}=466\right.$ Pa.s $), b$ is a parameter which balances the upper convected derivative and the Jauman derivative of the extrastress tensor $\tau_{i}[28](b=0.15), p$ is the isotropic part of the stress tensor $\sigma$ and $\dot{\epsilon}(u)$ is the rate of deformation tensor. $\eta_{i}$ and $\lambda_{i}$ are deduced from the dynamic rheology measurements using Marquadt-Levenberg method (table 1). Six modes allow to obtain a good agreement with dynamic measurements [29].

The figures 3 and 4 shows that the viscosity and relaxation time of PET1 are shear dependant. Therefore the Reynold number and the Weissenberg number can be defined by describing this dependency by a power law ( $\eta=$ $\eta_{0}|\dot{\bar{\gamma}}|^{m-1}$ and $\lambda=\lambda_{0}|\dot{\bar{\gamma}}|^{n-1}$ for example). In this way, one gets for a die with a width $W$ and a thickness $h$ subjected to a flow rate $Q$, the following expressions

$$
R e=\rho \frac{Q^{2-m} h^{m-1} W^{m-2}}{\eta_{0}} \quad ; W e=\lambda_{0}\left(\frac{Q}{W h^{2}}\right)^{n}
$$




\section{Coextrusion experimental measurements}

Several experiments have been performed at Arcelor Mittal facilities in Thionville (France). The temperature regulation of both single screw extruders (diameter $25 \mathrm{~mm}$ and $30 \mathrm{~mm}$ respectively) and their rotation velocities have been adjusted in such a way to obtain a constant temperature of $260^{\circ} \mathrm{C}$ at die outlet. The die temperature has been also fixed to $260^{\circ} \mathrm{C}$. The rotation velocity of the two extruders has been varied and, after stabilization of the process, the quality of the coextruded sample has been quantified from 0 (good quality) to 5 (bad quality). In table 2 experimental observations are reported. A pressure sensor is put at the output of each extruder and measurements are made with and without the coat hanger die for various flow rates. In this way the pressure loss inside coextrusion die can be evaluated. We have kept samples for which the defects are not too important and correspond to rather regular waves. We assume that such disturbances could be computed more easily and exhibit a linear spatial growth rate.

\section{3D monolayer temperature dependent computation in the ex- trusion die}

To analyse the flow pattern, we perform a 3D monolayer calculation of the polymer flow in the coat hanger die using the finite element software REM3D (C) dedicated to polymer processing applications in injection and extrusion [30]. We consider the flow of PET1 with a purely viscous temperature dependent behaviour using the CarreauYasuda law (1). An anisotropic mesh with 55739 elements and 315314 nodes is used to mesh the computational domain depicted in figure 2. It is refined in the thickness and coarse in the two other directions [31]. We solve iteratively the mass and force balance equations (incompressible) and the energy balance equation till convergence. The computation is made for a flow rate $Q=1.10 \mathrm{~cm}^{3} / \mathrm{s}$ and the computed pressure loss is $3.4 \mathrm{MPa}$. This latter value is consistent with experimental measurement pressure loss $(3.5 \mathrm{MPa})$.

Figure 5 shows the velocity distribution in the die. The velocity component in the transverse direction is negligible except in the coat hanger region which distributes the polymer throughout the width of the die (figure 5b). As a consequence, the flow may be considered as $2 \mathrm{D}$ both in the inlet and in the outlet regions of the die. It is possible to compute a crude evaluation of the Reynolds and Weissenberg numbers in this two zones for both polymers 
(see relation (4)). The Reynolds number is around $10^{-5}$ for both fluids and both zones. For PET1, the Weissenberg number is around 1 in inlet zone and larger in the outlet zone. It increases with the shear rate (or the mean flow).

Figure 6 shows the mean temperature field (throughout the die gap) when the initial temperature of the polymer is $281^{\circ} \mathrm{C}$ and the temperature of the die is $260^{\circ} \mathrm{C}$. One observes an important temperature decrease within the die, but the flow may be considered as isothermal both in the inlet and the outlet regions.

We assume that introducing a viscoelastic constitutive equation will provide the same conclusions for the velocity field and the temperature field. As a consequence we will develop 2D dynamic isothermal viscoelastic computations in these two flow regions in order to analyse if perturbations are damped or amplified.

\section{2D multilayer viscoelastic isothermal computation}

We apply the finite element viscoelastic computation method, already developed by Silva et al. [32, 33], to the multi-mode Johnson-Segalman model (2-3). The numerical method is explained for one layer and for one mode. First the dynamic equilibrium and mass balance equations are solved by a splitting method which writes when neglecting mass and inertia forces:

$$
\begin{aligned}
\nabla \cdot\left[2 \eta_{s} \dot{\epsilon}\left(\mathbf{u}_{n+1}\right)\right]-\nabla p_{n+1} \mathbb{I} & =\nabla \cdot \tau_{n} \\
\nabla \cdot u_{n+1} & =0
\end{aligned}
$$

At time step $(n+1)$ one solves equations (5) with a known value of the extra-stress $\tau_{n}$ at time step $n$. Then one solves the constitutive equation (6) to determine $\tau_{n+1}$ :

$$
\tau_{n+1}+\lambda\left(\left(1-\frac{b}{2}\right) \frac{\delta \tau_{n+1}}{\delta t}+\frac{b}{2} \frac{D \tau_{n+1}}{D t}\right)=2 \eta \dot{\epsilon}\left(\mathbf{u}_{n+1}\right)
$$

An advantage of decoupling is that the global problem, with a large number of unknowns, and therefore large-consuming memory, is broken up into smaller systems: a Stokes incompressible problem perturbed by an elastic term (treated as a known body force term) and the non-linear constitutive equations that allow the determination of $\tau$. Furthermore, each problem can 
be treated taking into account its mathematical type: the Stokes problem (5) is elliptic and linear, whereas the computation of extra stress tensor with the constitutive equation (6) involves non-linearities and convection. Disadvantages are only related to the need of a marching time scheme for both calculations, which could lead to time instabilities if not carefully carried out. Consequently the computational time is rather important as we have to deal with two constraints on the time step $\Delta t$ :

$$
\Delta t<1 / \max (\dot{\bar{\gamma}}) \quad \text { and } \quad \Delta t<\min _{i}\left(\lambda_{i}\right)
$$

Finally, the time step chosen in the sequel is $\Delta t=.005$. Finite element method is used to solve the above equations. For the Stokes equation (5), we choose a linear interpolation for velocity and pressure with bubble enrichment for velocity, the $P_{1}^{+} / P 1$ element, also referred as the MINI-element [34]. Note also that a solvent viscosity is included in the proposed formulation (5). Some authors can completely eliminate it, by using stabilization methods like the EVSS or the DEVSS formulations. In our approach, solvent viscosity can be very small because the bubble stabilization procedure is approaching the DEVSS method of Guénette and Fortin [35].

The evolution equation (6) is solved through a Space-Time Discontinuous Galerkin method. Basically, Space-Time means that it allows a simultaneous resolution in space and in time $(x, t)$ and the computational domain is $(\Omega \times] 0, t_{e}[)$, where $t_{e}$ is the duration of the process studied; Discontinuous Galerkin means that we choose discontinuous interpolations both in space (low order) and in time (higher order). On the element $K$, extra stress is interpolated by functions constant and discontinuous in space, $P 0(K)$, and polynomial (degree $n)$ and discontinuous in time $\left(P n(] t_{i}, t_{i+1}[)\right)$. The case $n=0$ corresponds to the classical discontinuous Galerkin method [36, 37].

For the bi-layer coextrusion considered here (figure 8), the bottom layer $\Omega_{1}$ is viscoelastic and the upper layer $\Omega_{2}$ is purely Newtonian and direct solutions of equations (5) are computed. In addition to classical sticking conditions along the die walls, we assume at the interface $\Gamma$ the continuity of the velocity, the non-miscibility and the continuity of the normal stress components (neglecting surface tension) :

$$
\mathbf{u}^{1}=\mathbf{u}^{2} ; \mathbf{u} \cdot \mathbf{n}=0 ; \sigma^{1} \cdot \mathbf{n}=\sigma^{2} \cdot \mathbf{n}
$$

where $\mathbf{n}$ is the normal at the interface. Two flow rates are imposed at the inlet (one for each polymer) and a free surface condition at die exit. The 
first conditions correspond to a Dirichlet conditions as the imposed velocity is chosen such that it satisfies the condition

$$
\int_{\Gamma} \mathbf{u}^{i} \cdot \mathbf{n} d \Gamma=Q_{i}
$$

in each layer, $\mathbf{n}$ being the normal to die inlet. The second condition is the classical Neuman boundary condition, that is

$$
\sigma^{i} \cdot \mathbf{n}=0
$$

$\mathbf{n}$ being here the normal to die outlet.

In order to calculate a moving free surface, we have adopted an Eulerian approach. Let us introduce $\mathbb{I}$ the characteristic function of the first layer domain defined by:

$$
\mathbb{I}(x, t)= \begin{cases}1 & \text { if } x \in \Omega_{1} \\ 0 & \text { if } x \notin \Omega 1\end{cases}
$$

In this way, the rheological behaviour of both fluids depends on this characteristic function and the finite element formulation satisfies automatically the velocity and normal stress component continuity at the interface. The non-miscibility (kinematic) condition which gives the evolution of each phase with respect to flow motion corresponds to a transport equation for the characteristic function [38]:

$$
\frac{\partial \mathbb{I}}{\partial t}+\mathbf{u} \cdot \nabla \mathbb{I}=0
$$

Therefore, the characteristic function itself becomes an unknown function which can be approximated using a finite element technique. In practical, an approximation $\mathbb{I}^{h}$ constant by element $\left(P_{0}\right.$ approximation) has been used:

$$
\left.\mathbb{I}^{h}\right|_{K}=\mathbb{I}^{K}=\frac{|K| \cap\left|\Omega_{1}\right|}{|K|}
$$

It is simply the ratio in each element $K$ between the surface occupied by the phase 1 and the total surface of element $K$. This hyperbolic equation is also solved by a space-time Galerkin Discontinuous method [39]. In order to improve the precision, refined anisotropic meshes have been used around the interface (see figure 7) [31].

Post-processor tools allow to visualize and analyse precisely the evolution of the interface and are described in the sequel. First, the thickness of the 
bottom layer at each abscissa $x_{i}$ is given by:

$$
h\left(x_{i}\right)=\int \mathbb{I}\left(y, x_{i}\right) d y=\sum_{K} \mathbb{I}^{K} l_{K}\left(x_{i}\right)
$$

where $l_{K}\left(x_{i}\right)$ is the length of the vertical line $\left(x_{i}, y\right)$ which belongs to element $K$ (figure 7 ).

For a more comprehensive view of disturbance evolution, a xt diagram is plotted. This diagram shows the isovalue of relative perturbation in the $(x, t))$ plane. Computations are made for a large number of time steps (typically ten time the forcing period) and the mean value of interface is estimated for each abscissa $x_{i}$ :

$$
h_{m}\left(x_{i}\right)=\sum_{j=1}^{N_{t}} h\left(x_{i}, t_{j}\right) / N_{t}
$$

and therefore the relative perturbation writes:

$$
\partial h\left(x_{i}, t_{j}\right)=\frac{h\left(x_{i}, t_{j}\right)-h_{m}\left(x_{i}\right)}{h_{m}\left(x_{i}\right)}
$$

As shown in figures 9 and 10, travelling wave gives isovalues parallel to a line satisfying the relation

$$
2 \pi t / \lambda_{t}-2 \pi x / \lambda_{x}=C t e
$$

$\lambda_{t}$ and $\lambda_{x}$ being the temporal and spatial periods of the travelling wave. Spatial evolution is represented by color evolution along $x$ for each time $t$. Finally, the numerical value of growth or decay rate of the disturbance is computed by tracing the exponential envelope. The spatial and temporal periods of the progressive wave are computed with a two dimensional Fourier analysis.

\section{2D isothermal Dynamic stability analysis}

For purely viscous fluids, the driving force for instability development is the Reynolds number. Due to the high viscosity of both polymers, the Reynolds number is very small (of the order $10^{-5}$ ) and Mahdaoui showed [40] that it was unable to develop instabilities. In the present situation the 
driving force is the Weissenberg number which is near zero for PET2 but may be of the order of one for PET1. Figures 8 present the stationary interfaces for both inlet (figure 8a) and outlet (figure 8b) zones for the case $\mathrm{N}^{o} 4$ of table 1 . In the inlet region, the interface between both fluids stabilizes on a short distance (less than one gap) and then remains flat. The same situation is observed in the outlet region. The location of the interface changes only in the convergent part of the die and stabilizes again.

Starting from the stable interface location (Figures 8a and 8b), a periodic disturbance is applied on the first layer [40]:

$$
Q_{1}=Q_{10}\left(1+A \sin \left(\frac{2 \pi}{T} t\right)\right)
$$

where the amplitude of the perturbation $A=0.1$ and $T$ is the forcing period which may correspond to experimental flow fluctuations related to the screw rotation of each extruder. This would correspond to forcing frequencies between 0.3 and $1.5 \mathrm{~Hz}$ and we have chosen to check two frequencies $0.5 \mathrm{~Hz}$ and $1 \mathrm{~Hz}$. Diagrams xt show the appearance of a travelling wave. Moreover the computations of the growth rate are made when they can be fitted by an exponential law ( that means that it does not correspond to a non linear saturation). The sign of this coefficient gives the stability. For more complex situation, we only check if the perturbation is bigger at the exit than at the entrance.

\subsection{Numerical simulations in the inlet zone}

The inlet zone corresponds to a rectangular channel of size $110 \times 10$ (aspect ratio $\sim .091)$. Figures 9 show the evolution of the perturbation along the inlet zone of the die for $0.5 \mathrm{~Hz}$ forcing frequency and experimental case number 4 (see table 1 ). It is found that the perturbation has the form of travelling wave having a damping amplitude and a temporal dependency corresponding to the forcing frequency. The spatial periodicity is $23 \mathrm{~mm}$ and the decay rate of the disturbance is 0.007 . The values for the other cases are reported in table 3 and show that the perturbation is always damped. The forcing frequency is always recovered expect for the case $\mathrm{N}^{o} 2$. In this case, the spatial disturbance associated to forcing frequency is too large with respect to die length. Therefore the system selects a smaller spatial periodicity that induces a slight difference between the measured and imposed temporal frequencies. 


\subsection{Numerical simulation in the outlet zone}

The outlet zone corresponds to two successive rectangular channels (60 $\times 2$ and $17 \times .9)$ connected by a convergent geometry with an angle of $15^{\circ}$ (see figure $8 \mathrm{~b}$ ). Figures 10 show the evolution of the perturbation along this outlet zone for $0.5 \mathrm{~Hz}$ forcing frequency and experimental case number 4 (see table 1). Here again, the perturbation has the form of a travelling wave but it is amplified. The spatial evolution for $1 \mathrm{~Hz}$ forcing frequency is also plotted in figure 11. The situation is less unstable but this picture could help the discussion and the comparisons when processing conditions will be changed. The xt diagram shows two areas before and after the convergent with different spatial periodicities (see figures 10). Figure 10a shows that the perturbation is amplified in the first flow region and not damped by the convergent part and the final flow region.

The two dimensional Fourier analysis and the damping (or growth) rate of the perturbation is computed before the convergent part. The final die geometry is too short to allow calculating an accurate damping or growth rate factor in this region. In the case depicted in figures 10, the forcing frequency is recovered. There are various situations for which the growth rate is difficult to evaluate:

- For case $\mathrm{N}^{o} 1$ and forcing frequency $0.5 \mathrm{~Hz}$, the perturbation is damped in the first part and it is amplified in the convergent part.

- For case $\mathrm{N}^{o} 1$ and forcing frequency $1 \mathrm{~Hz}$, several frequencies are activated and it is difficult to compute a growth rate as the disturbance is subjected to nonlinear effects. Nevertheless the perturbation remains important at the die exit and the configuration is labelled as unstable.

- For case $\mathrm{N}^{o} 4$ and forcing frequency $1 \mathrm{~Hz}$, the perturbation is first spatially amplified, then is damped just before the convergent part. The final part does not seem to change the amplitude of the perturbation.

Finally the conclusions of this section are the following :

- The perturbation is only amplified in the outlet zone.

- The numerical computations are rather in good agreement with experiments: stable samples (case 2 and 3 ) reported in table 2 correspond to negative growth rate of the perturbations. The most unstable sample 
(case 1) reported in table 2 is also reported as unstable for both frequencies in table 4 . Sample 4 which is reported as slightly unstable in table 2 is reported as stable for hight frequency $(1 \mathrm{~Hz})$ and unstable for low frequency $(0.5 \mathrm{~Hz})$ in table 4 .

- The small frequencies are most dangerous for this system.

- The influence of the convergent geometry is not clear: it reduces the amplitude of perturbations for case 4 (forcing frequency $1 \mathrm{~Hz}$ ) but amplified it in case 1 (forcing frequency $0.5 \mathrm{~Hz}$ ).

\section{Other Processing Conditions (Geometry, Flow rate, Tempera- ture)}

In this section, we analyse the influence of processing conditions and die outlet geometry on the appearance of perturbation. For a fixed thickness ratio (which is imposed by the target sheet application), it is possible to modify both flow rates as well as the temperature of one or both polymers in order to prevent the appearance of wavy interface. Case $\mathrm{N}^{o} 4$ in the outlet zone is considered for different flow rates and different temperatures.

First, the flow rates of both polymers are multiplied by two which means that the shear rate and viscosity ratios change. Figure 12 depicts the spatial evolution of the disturbance for the two forcing frequencies 0.5 et $1 \mathrm{~Hz}$. In both cases, the travelling waves have a spatial amplification. It is most important for $1 \mathrm{~Hz}$ (the growth rate is around 0.02) than $0.5 \mathrm{~Hz}$ (growth rate $\sim 0.015)$. Note that other spatial frequencies are observed which characterizes a nonlinear behaviour or saturation. In that case we are faced to two contradictory phenomena: increasing the flow rates and so the shear rates will decrease the viscosity ratio between the two polymers (see figures 2 ) and this is supposed to stabilize the process, but at the same time the Weissenberg number which drives the development of the instability will increase.

The computations of section 6 are made with a temperature of $260^{\circ} \mathrm{C}$. If the temperature is increased to $280^{\circ} \mathrm{C}$, the viscosity ratio is increased because the activation energy are different $(38 \mathrm{KJ} / \mathrm{mol}$ for the PET1 and 61 $K J / m o l$ for the PET2). In the same time the relaxation time of PET1 (the distribution of relaxation time of table 1) decreases and also the Weissenberg number.

Figures 13 show spatially amplified travelling waves for both forcing frequencies. For $0.5 \mathrm{~Hz}$, the growth rate is 0.01 , whereas it is 0.006 for $1 \mathrm{~Hz}$. 
They are smaller than those computed for $260^{\circ} \mathrm{C}$ (see table 4). Here again, increasing the Weissenberg number seems to influence more the stability of process than increasing the viscosity ratio.

We investigate the influence of the convergent angle between the two slit flows on the amplitude of the perturbation. Three geometries have been considered with $0^{\circ}$ (parallel slit die), $15^{\circ}$ (previous slit die) and $30^{\circ}$.

For a slit of uniform thickness $\left(0^{\circ}\right.$ angle), figure 14 shows the evolution of perturbations along the flow direction for the forcing frequencies 0.5 and $1 \mathrm{~Hz}$. The generated travelling waves are maintained for frequency $1 \mathrm{~Hz}$ and amplified for $0.5 \mathrm{~Hz}$. Therefore without the convergent geometry, the perturbation is maintained or amplified whereas it was slightly damped for forcing frequency $1 \mathrm{~Hz}$ and amplified for $0.5 \mathrm{~Hz}$ for the angle $15^{\circ}$ which was previously investigated.

For the angle $30^{\circ}$, figures 15 show the evolution of perturbations along the flow motion for the forcing frequencies 0.5 and $1 \mathrm{~Hz}$. As previously the initial perturbation is amplified for $0.5 \mathrm{~Hz}$ and maintained for $1 \mathrm{~Hz}$. The change of spatial periodicity after the convergent geometry is pointed out clearly. It is more important than for an angle of $15^{\circ}$. Then, the convergent geometry modifies the spatial periodicity of perturbation without amplification. Khomami and Wilson [41] studied experimentally interfacial instabilities in both diverging and converging channels. They found that it is less amplified in converging channel. Theoretical analysis of Hinch and coworkers [42] predicts the stabilizing effect of converging channel flows and a destabilizing effect for diverging ones. In this industrial set-up , we do not observed any significant influence of the shape of the convergent geometry on the development of instabilities.

\section{Conclusions}

Dynamic stability analysis of the interface between two layers in the coextrusion process of thermoplastic polymers has been performed. It points out the development of progressive waves along the die geometry. For viscoelastic fluids, the driving force is the Weissenberg number which is much higher than the Reynolds number. The results show that the amplitude of the progressive waves may increase or damp towards the die exit depending on the processing parameters, the die geometry and the rheology of the polymer. The results are in agreement with experiments done on industrial coextrusion device. Nevertheless they require further experiments and the 
test of more advanced constitutive equations to become an useful tool for optimizing the processing parameters (temperatures, output rates, ...), the die geometry and then adjusting the rheology of the coextruded polymers.

\section{Acknowledgements}

O. Mahdaoui has received a support from Arcelor-Mittal. The authors

also thank A. Merveille from Arcelor-Mittal for his helpful comments and remarks.

\section{References}

[1] C. Han, Y. Kim, H. Chin, Rheological investigation of interfacial instability in two layer flat-film coextrusion, Polym. Eng. Rev. 4 (1984) $177-217$.

[2] C. Han, R. Shetty, Studies on multilayer film coextrusion I. The rheology of flat film coextrusion, Polym. Eng. Sci. 16 (1976) 697-705.

[3] C. Han, R. Shetty, Studies on multilayer film coextrusion II. Interfacial instability in flat film coextrusion, Polym. Eng. Sci. 18 (1978) 180-186.

[4] N. Anturkar, T. Papanastasiou, J. Wilkes, Estimation of critical stability parameters by asymptotic analysis in multilayer extrusion, Polym. Eng. Sci. 33 (1993) 1532-1539.

[5] R. Valette, P. Laure, Y. Demay, J.-F. Agassant, Investigation of the interfacial instabilities in the coextrusion flow of polyethylene and polystyrene, Int. Polym. Process. XVIII-2 (2003) 171-178.

[6] R. Valette, P. Laure, Y. Demay, J.-F. Agassant, Experimental investigations of the development of interfacial instabilities in two-layer coextrusion dies, Int. Polym. Process. XIX-2 (2004) 118-128.

[7] G. Wilson, B. Khomami, An experimental investigation of interfacial instabilities in multilayer flow of viscoelastic fluids. Part I. Incompatible polymer systems, J. Non-Newtonian Fluid Mech. 45 (1992) 355-384. 
[8] G. Wilson, B. Khomami, An experimental investigation of interfacial instabilities in multilayer flow of viscoelastic fluids. II. Elastic and nonlinear effects in incompatible polymer systems, J. Rheol. 37 (1993) 315-339.

[9] G. Wilson, B. Khomami, An experimental investigation of interfacial instabilities in multilayer flow of viscoelastic fluids. III. Compatible polymer systems, J. Rheol. 37 (1993) 341-354.

[10] B. Khomami, M. Ranjbaran, Experimental studies of interfacial instabilities in multilayer pressure-driven flow of polymeric melts, Rheol. Acta 36 (1997) 345-366.

[11] B. Khomami, K. Su, An experimental/theoretical investigation of interfacial instabilities in superposed pressure-driven channel flow of Newtonian and well characterized viscoelastic fluids. part I: linear stability and encapsulation effects, J. Non-Newtonian Fluid Mech. 91 (2000) 59-84.

[12] R. Valette, P. Laure, Y. Demay, J.-F. Agassant, Convective linear stability analysis of two-layer coextrusionflow for molten polymers, J. Non Newtonian Fluid Mech. 121 (2004) 41-53.

[13] R. Valette, P. Laure, Y. Demay, A. Fortin, Convective instabilities in coextrusion process, Int. Polym. Process. XVI-2 (2001) 192-197.

[14] M. Gaster, A note on the relation between temporally-increasing and spatially-increasing disturbances in hydrodynamic stability, J. Fluid Mech. 14 (1962) 222-224.

[15] K. Chen, Interfacial instability due to elastic stratification in concentric coextrusion of two viscoelastic fluids, J. Non-Newtonian Fluid Mech. 40 (1991) 155-175.

[16] Y. Su, B. Khomami, Interfacial stability of multilayer viscoelastic fluids in slit and converging channel dies geometries, J. Rheol. 36 (1992) 357387.

[17] Y. Su, B. Khomami, Purely elastic interfacial instabilities in surperposed flow of polymeric fluids, Rheol. Acta 31 (1992) 413-420. 
[18] P. Laure, H. L. Meur, Y. Demay, J. Saut, S. Scotto, Linear stability of multilayer plane Poiseuille flows of Oldroyd-B fluid, J. Non-Newtonian Fluid Mech. 71 (1997) 1-23.

[19] H. Ganpule, B. Khomami, The effect of transient viscoelastic properties on interfacial instabilities in superposed pressure driven channel flows, J. Non-Newtonian Fluid Mech. 80 (1999) 217-249.

[20] H. Ganpule, B. Khomami, An investigation of interfacial instabilities in the superposed channel flow of viscoelastic fluids, J. Non-Newtonian Fluid Mech. 81 (1999) 27-69.

[21] Y. Demay, Computation of 2D viscoelastic flows for differential constitutive equation, volume 5, Elsevier, pp. 237-255.

[22] F. Baaijens, An iterative solver for the DEVSS/DG method with application to smooth and non-smooth flows of the Upper Convected Maxwell fluid, J. Non-Newtonian Fluid Mech. 75 (1998) 119-138.

[23] H. Yamaguchi, A. Mishima, T. Yasumoto, T. Ishikawa, An arbitrary Lagrangian-Eulerian approach for simulating viscoelastic fluids, J. NonNewtonian Fluid Mech. 80 (1999) 251-272.

[24] M. Zatloukal, C. Tzoganakis, J. Vlcek, P. Saha, Numerical simulation of polymer coextrusion flows: a criterion for detection of ?wave? interfacial instability onset, Int. Polymer Processing XVI (2001) 198-207.

[25] D.W. Bousfield, R. Keunings, G. Marrucci, M.M. Denn, Non-linear analysis of the surface tension driven breakup of viscoelastic filaments, J. Non-Newtonian Fluid Mech 21 (1986) 79-97.

[26] F. Langouche, B. Debbaut, Rheological characterisation of a highdensity polyethylene with a multi-mode differential viscoelastic model and numerical simulation of transient elongational recovery experiments, Rheol. Acta 38 (1999) 48-64.

[27] M. Johnson, D. Segalman, A model for viscoelastic fluid behavior which allows nonaffine deformation, J. Non-Newtonian Fluid Mech. 2 (1977) 255-270. 
[28] M. Johnson, D. Segalman, A model for viscoelastic fluid behavior which allows non-affine deformation, J. Non-Newtonian Fluid Mech. 2 (1992) $255-270$.

[29] G. Boukellal, A. Durin, R. Valette, J.-F. Agassant, Evaluation of a tubebased constitutive equation using conventional and planar elongation flow optical rheometers, Rheologica Acta 50 (2011) 547-557.

[30] L. Silva, J. Agassant, T. Coupez, Three-dimensional injection molding simulation, In Injection molding, technology and fundamentals, Eds M.R. Kamal, I.A. Isayev, S.J. Liu, Hanser (2009) 599-651.

[31] C. Gruau, T. Coupez, 3D tetrahedral, unstructured and anisotropic mesh generation with adaptation to natural and multidomain metric, Comput. Methods Appl. Mech. Engrg. 194 (2005) 4951-4976.

[32] L. Silva, Viscoelastic compressible flow and application in 3D injection modeling simulation, Ph.D. thesis, Ecole Nationale Supérieure des Mines de Paris, 2004.

[33] L. Silva, R. Valette, P. Laure, T. Coupez, A new three-dimensional mixed finite element for direct numerical simulation of compressible viscoelastic flows with moving free surfaces, Int. J. Mater. Form. 5 (2012) 55-72.

[34] D. Arnold, F. Brezzi, M. Fortin, A stable finite element for the Stokes equations, Calcolo 21 (1984) 337-344.

[35] R. Guénette, M. Fortin, A new finite element method for computing viscoelastic flows, J. Non-Newtonian Fluid Mech. 60 (1995) 27-52.

[36] B. Cockburn, C.-W. Shu, The local discontinuous Galerkin method for time-dependent convection-diffusion system, SIAM J. Numer. Anal. 35 (1998) 2440-2463.

[37] A. Fortin, A. Béliveau, M. Heuzey, A. Lioret, Ten Years Using Discontinuous Galerkin Method for Polymer Processing Problems, In Discontinuous Galerkin Methods : Theory, Computation and Applications, Springer (1998) 321-326. 
[38] A. Fortin, Y. Demay, J. Agassant, Computation of stationary interface between generalized Newtonian fluids, Revue Européenne des Eléments finis 1 (1992) 181-196.

[39] S. Batkam, J. Bruchon, T. Coupez, A space-time discontinuous Galerkin method for convection and diffusion in injection moulding, Int. J. Mater. Form. 7 (2004) 11-33.

[40] O. Mahdaoui, Étude numérique des Instabilités dans le procédé de coextrusion des polyesters, Ph.D. thesis, Ecole Nationale Supérieure des Mines de Paris, 2008.

[41] B. Khomami, G. Wilson, An experimental investigation of interfacial instability in superposed flow of viscoelastic fluids in a converging/diverging channel geometry, J. Non-Newtonian Fluid Mech. 58 (1995) 47-65.

[42] E. Hinch, O. Harris, J. Rallison, The instability mechanism for two elastic liquids being co-extruded, J. Non-Newtonian Fluid Mech. 43 (1992) 311-324. 


\begin{tabular}{|l|c|c|c|c|c|c|}
\hline$i$ & 1 & 2 & 3 & 4 & 5 & 6 \\
\hline \hline$\eta_{i}$ & 457.64 & 614.97 & 770.55 & 681.61 & 202 & 25.23 \\
$\lambda_{i}(s)$ & 0.04 & 0.14 & 0.51 & 1.84 & 5.44 & 20.37 \\
\hline
\end{tabular}

Table 1: Relaxation spectrum for PET1 at the reference temperature $260{ }^{\circ} \mathrm{C}$.

\begin{tabular}{|c|c|c|c|}
\hline case & PET1 $(\mathrm{rpm})$ & PET 2 $(\mathrm{rpm})$ & Experimental Observations \\
\hline 1 & 20 & 90 & 2 \\
2 & 50 & 25 & 0 \\
3 & 50 & 50 & 0 \\
4 & 50 & 90 & 1 \\
\hline
\end{tabular}

Table 2: Coextrusion observations: the quality of coextruded product has been marked from 0 (good quality) to 5 (very bad quality).

\begin{tabular}{|c|c|c|c|c|c||c|}
\hline Case & \multicolumn{2}{|c|}{$1 / \lambda_{t}(\mathrm{~Hz})$} & \multicolumn{2}{|c|}{$\lambda_{x}(\mathrm{~mm})$} & \multicolumn{2}{|c|}{ Growth rate } \\
& $1 \mathrm{~Hz}$ & $0.5 \mathrm{~Hz}$ & $1 \mathrm{~Hz}$ & $0.5 \mathrm{~Hz}$ & $1 \mathrm{~Hz}$ & $0.5 \mathrm{~Hz}$ \\
\hline \hline 1 & 1.0 & 0.50 & 12.23 & 6.80 & $-0.037(\mathrm{~S})$ & $-0.018(\mathrm{~S})$ \\
2 & 0.8 & 0.40 & 23.90 & 27.04 & $-0.009(\mathrm{~S})$ & $-0.004(\mathrm{~S})$ \\
3 & 1.0 & 0.50 & 9.05 & 18.20 & $-0.044(\mathrm{~S})$ & $-0.019(\mathrm{~S})$ \\
4 & 1.0 & 0.50 & 11.32 & 22.63 & $-0.035(\mathrm{~S})$ & $-0.007(\mathrm{~S})$ \\
\hline
\end{tabular}

Table 3: Fourier Analysis and growth rate of the perturbation in the inlet zone

\begin{tabular}{|c|c|c|c|c|c||c|}
\hline Case & \multicolumn{2}{|c|}{$1 / \lambda_{t}(\mathrm{~Hz})$} & \multicolumn{2}{c|}{$\lambda_{x}(\mathrm{~mm})$} & \multicolumn{2}{c|}{ Growth rate } \\
& $1 \mathrm{~Hz}$ & $0.5 \mathrm{~Hz}$ & $1 \mathrm{~Hz}$ & $0.5 \mathrm{~Hz}$ & $1 \mathrm{~Hz}$ & $0.5 \mathrm{~Hz}$ \\
\hline \hline 1 & 1.45 & 0.68 & 18.81 & 8.06 & $\mathrm{U}$ & $\mathrm{U}$ \\
2 & 1.00 & 0.52 & 3.52 & 7.05 & $-0.10(\mathrm{~S})$ & $-0.028(\mathrm{~S})$ \\
3 & 1.00 & 0.48 & 4.70 & 9.40 & $-0.043(\mathrm{~S})$ & $-0.002(\mathrm{~S})$ \\
4 & 1.06 & 0.50 & 6.27 & 14.7 & $\mathrm{~S}$ & $0.022(\mathrm{U})$ \\
\hline
\end{tabular}

Table 4: Fourier Analysis and growth rate of the perturbation in the outlet zone; the growth or damped rates are only given when the envelope of perturbation can be fitted by an exponential law. 


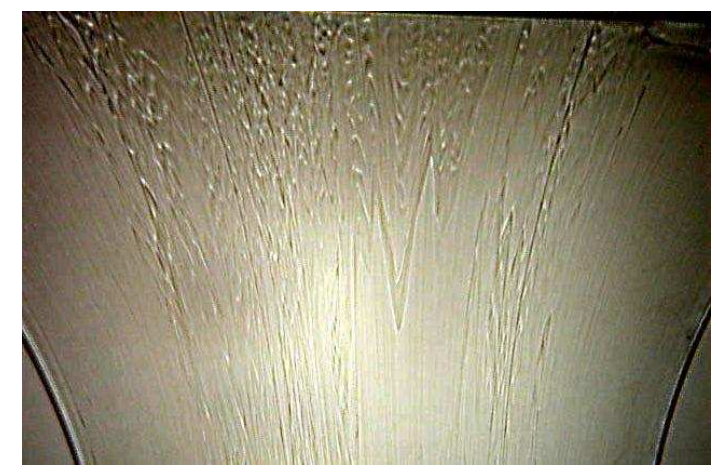

Figure 1: Characteristic defect on an industrial coextrusion line
first zone
second zone
third zone

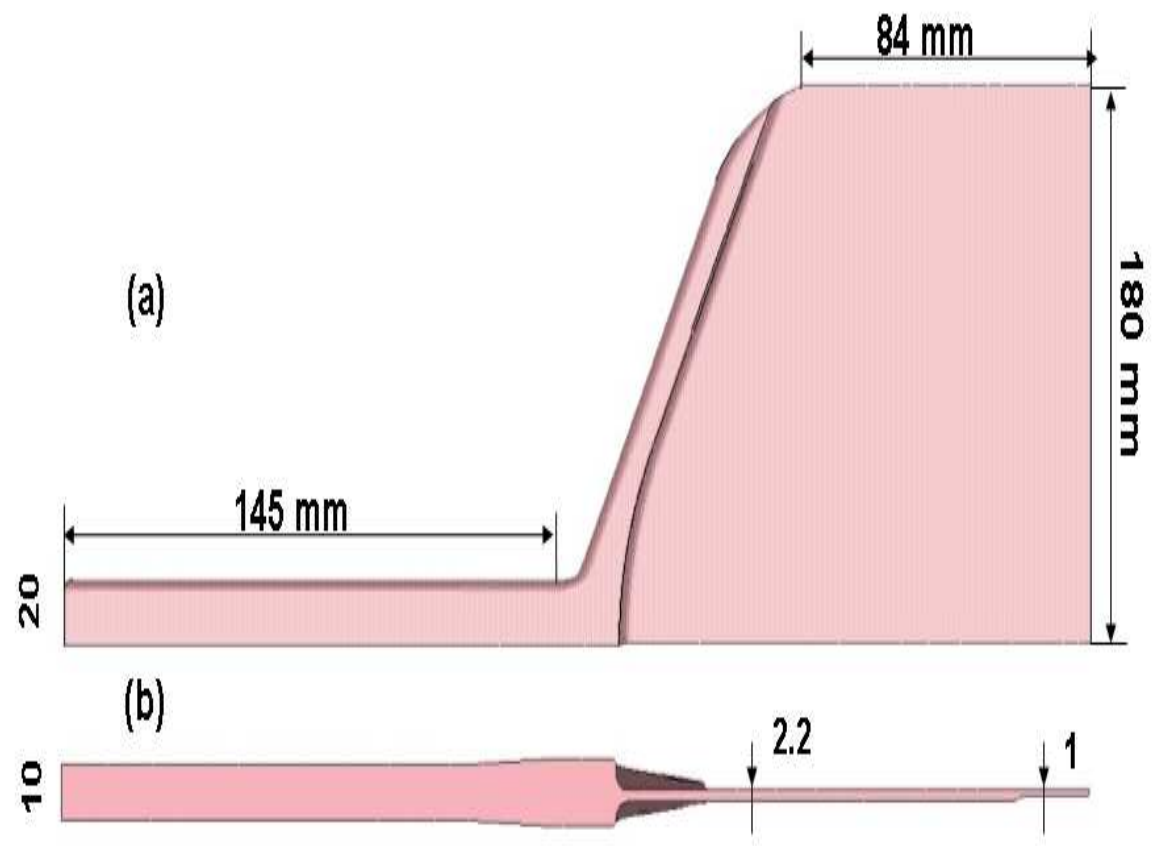

Figure 2: View from the top and through the thickness of the coat-hanger geometry: (a) $1 / 2$ die and the three different areas ; (b) view in the thickness direction along the symmetry plane 


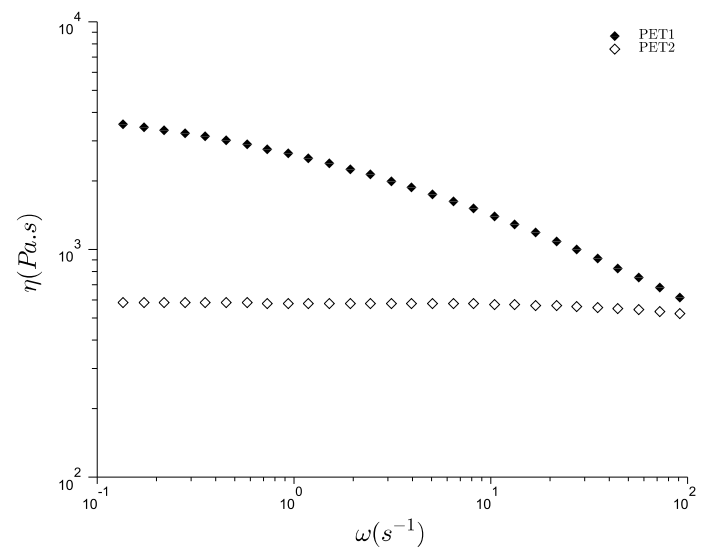

Figure 3: Viscosity as a function of frequency for both polyesters at $260{ }^{\circ} \mathrm{C}$ (deduced from complex modulus measurement on a cone and plate RMS 800 Rheometer)

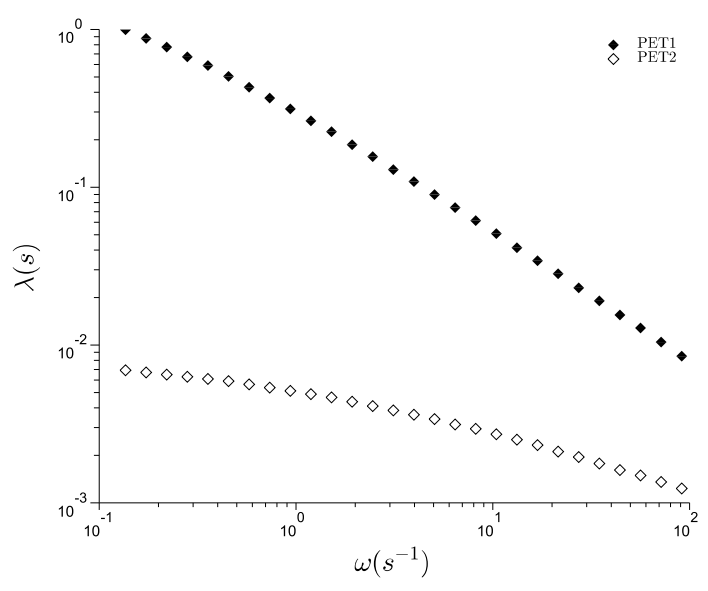

Figure 4: Mean relaxation time as a function of frequency (same protocol as in figure 3) 
a)

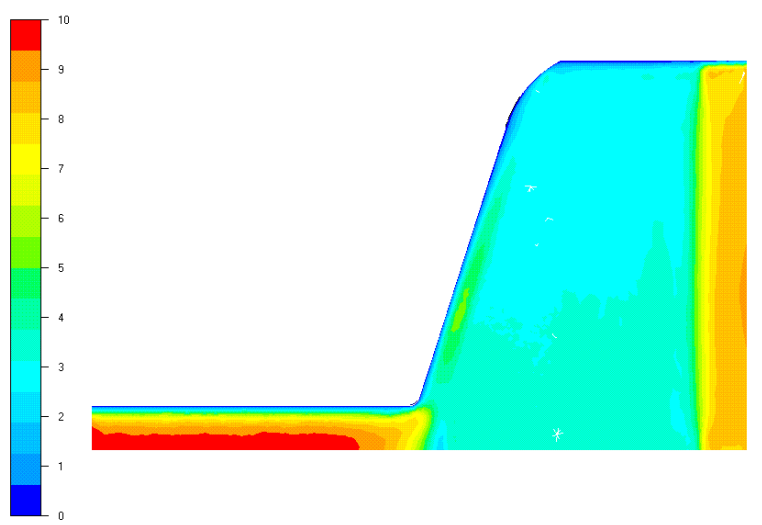

b)

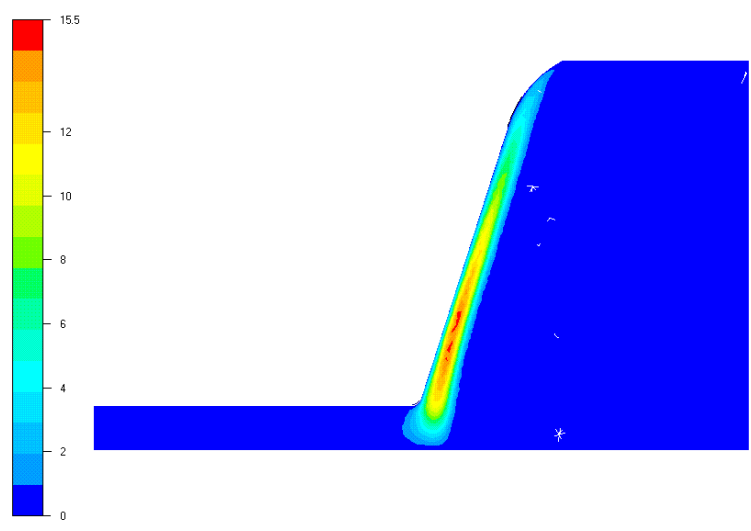

Figure 5: Mid-plane velocity field $(\mathrm{cm} / \mathrm{s})$ in the flow direction (a) and in the transverse direction (b) (the flow rate is $1.10 \mathrm{~cm}^{3} / \mathrm{s}$ ) and density $\rho=1.33 \mathrm{~kg} / \mathrm{dm}^{3}$. 


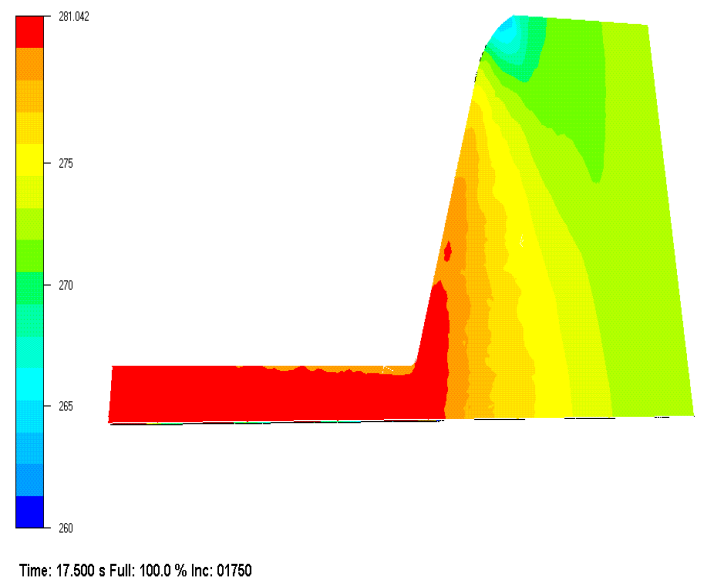

Figure 6: Mean temperature distribution in the die: the inlet temperature is $281^{\circ} \mathrm{C}$, die temperature is $260^{\circ} \mathrm{C}$; same flow rate as in figure 5
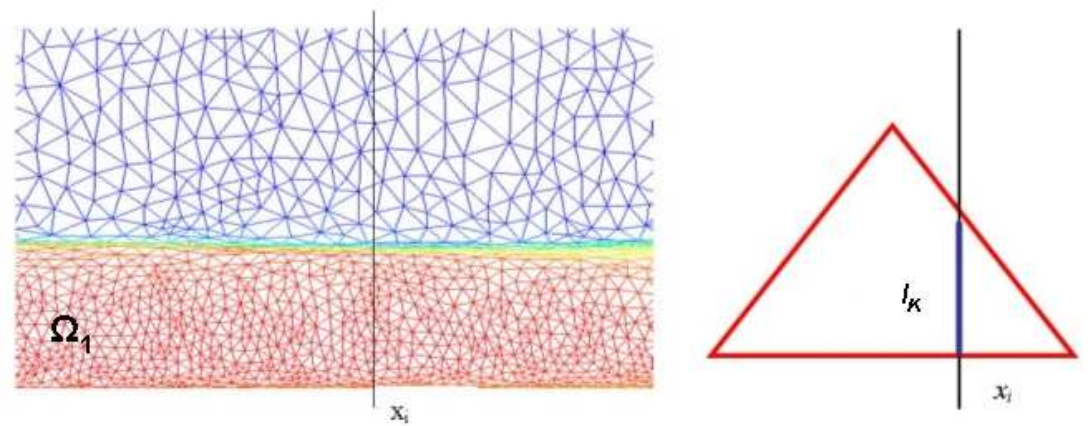

Figure 7: Isovalue of the characteristic function and mesh refining. Computation of the straight line $l_{K}$ which belongs to the element $K$ 
(a)

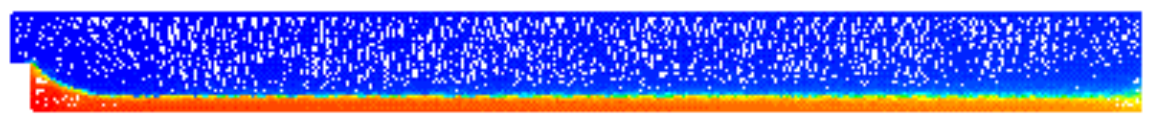

(b)

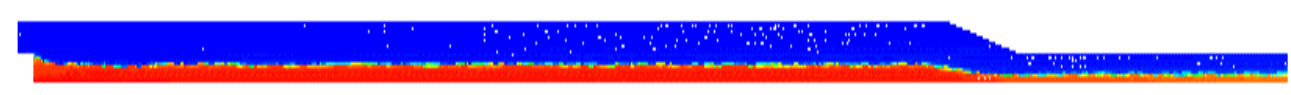

Figure 8: Calculation of stationary interface : (a) inlet zone; (b) outlet zone. ( $Q_{\text {PET1 }}=$ $1.99 \mathrm{~cm}^{3} / \mathrm{s} ; Q_{\text {PET2 }}=3.72 \mathrm{~cm}^{3} / \mathrm{s}$, case 4$)$ 
(a)

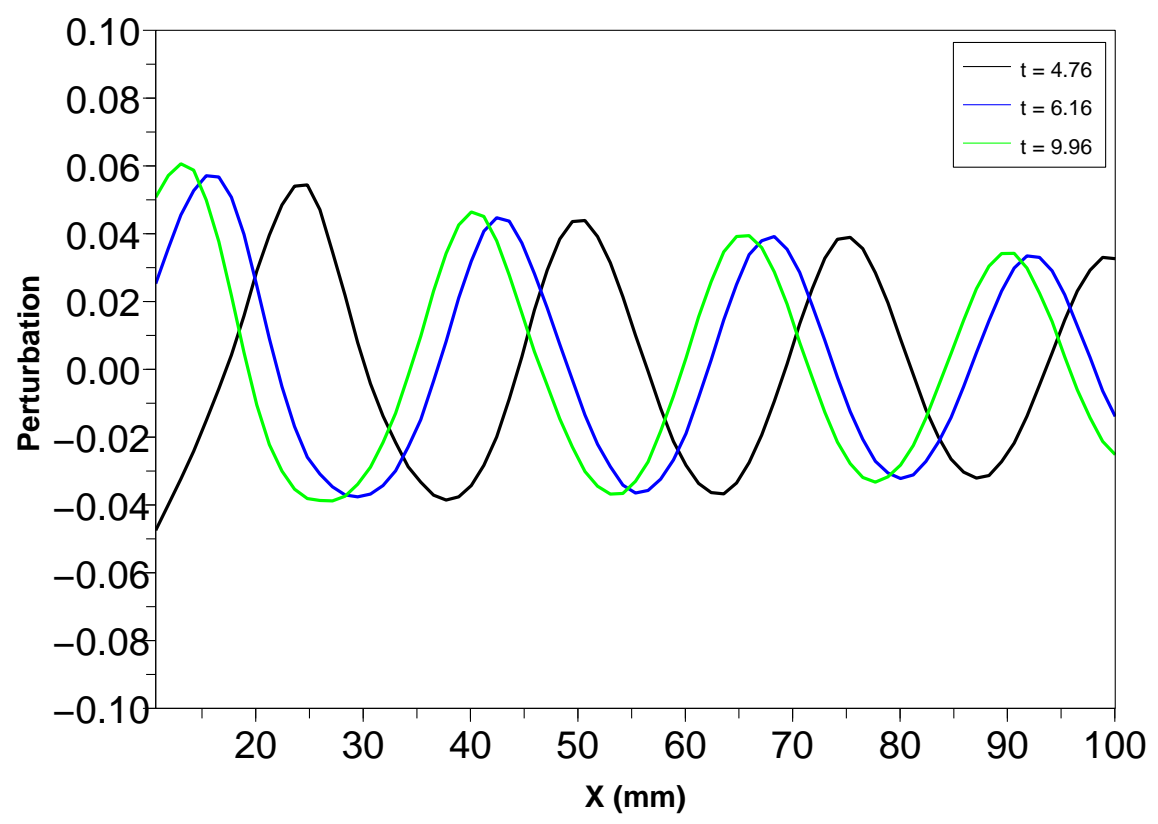

(b)

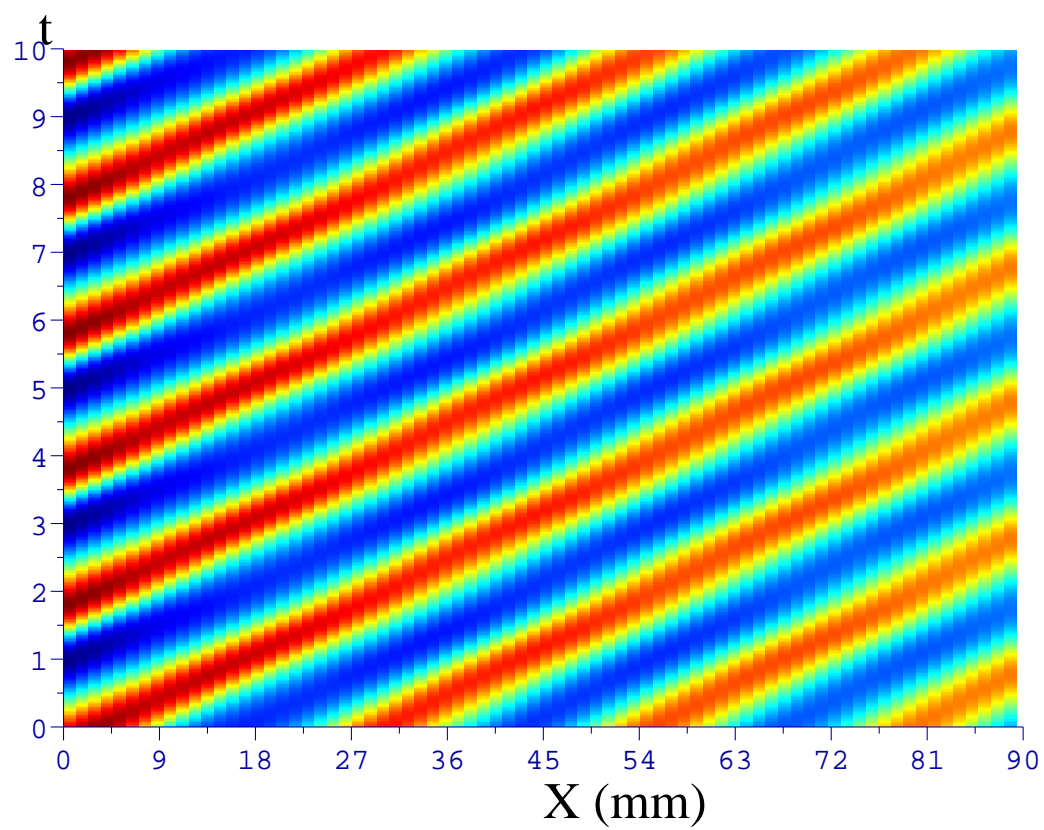

27

Figure 9: Relative perturbation for a forcing frequency of $0.5 \mathrm{~Hz}$ in the inlet zone; coextrusion conditions correspond to the unstable experimental case 4: (a) Spatial evolution at successive time steps; (b) xt diagram 
(a)

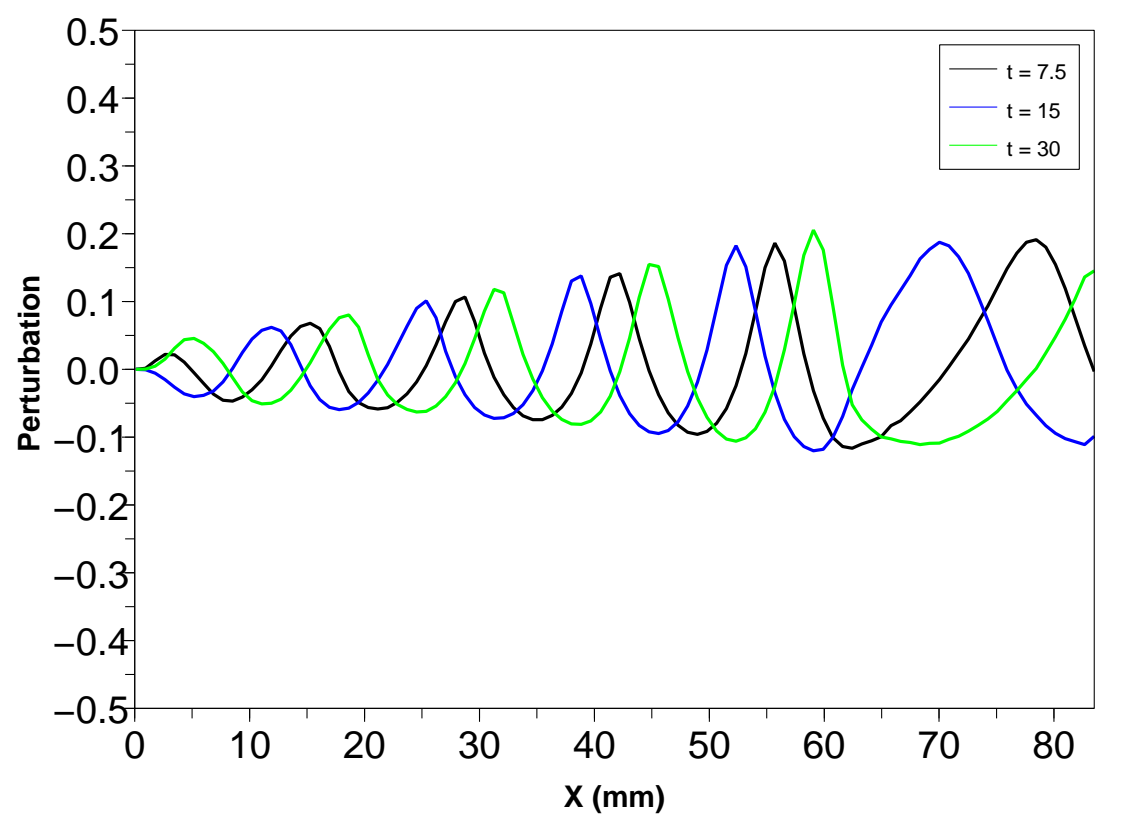

(b)

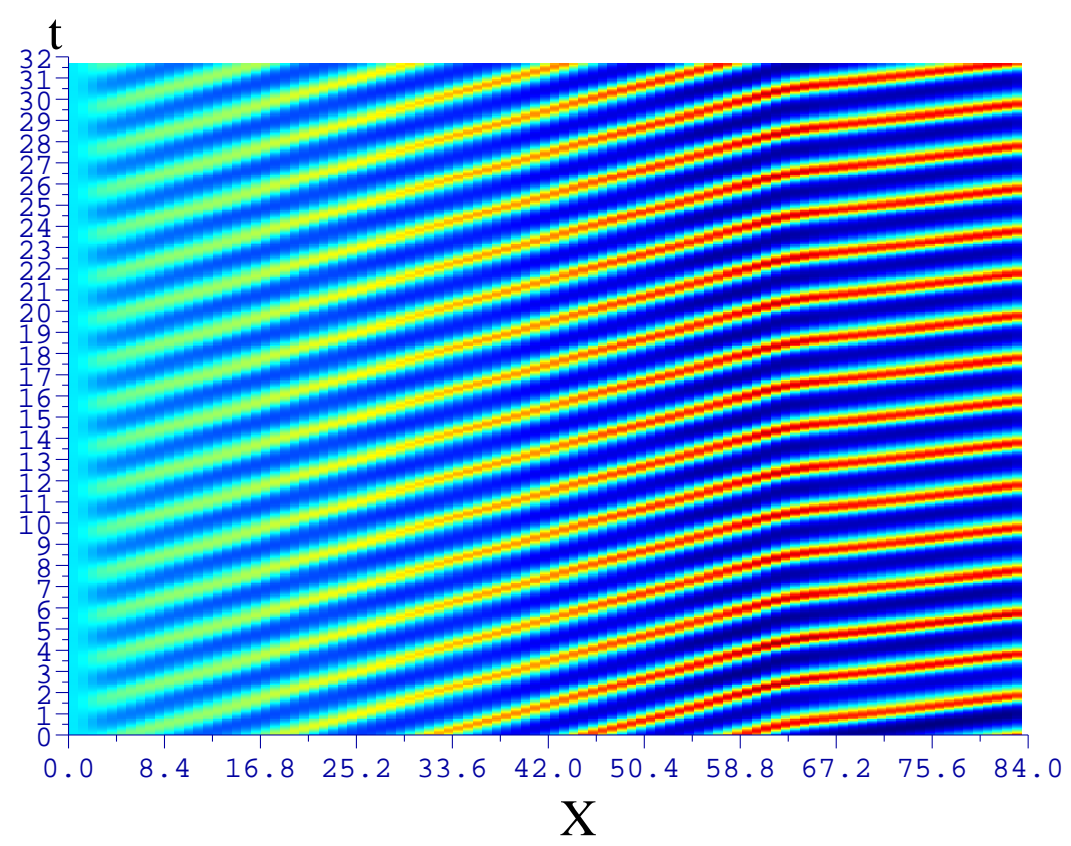

28

Figure 10: Relative perturbation for a forcing frequency of $0.5 \mathrm{~Hz}$ in the outlet zone; processing conditions correspond to the unstable experimental case 4: (a) Spatial evolution for various time steps; (b) xt diagram 


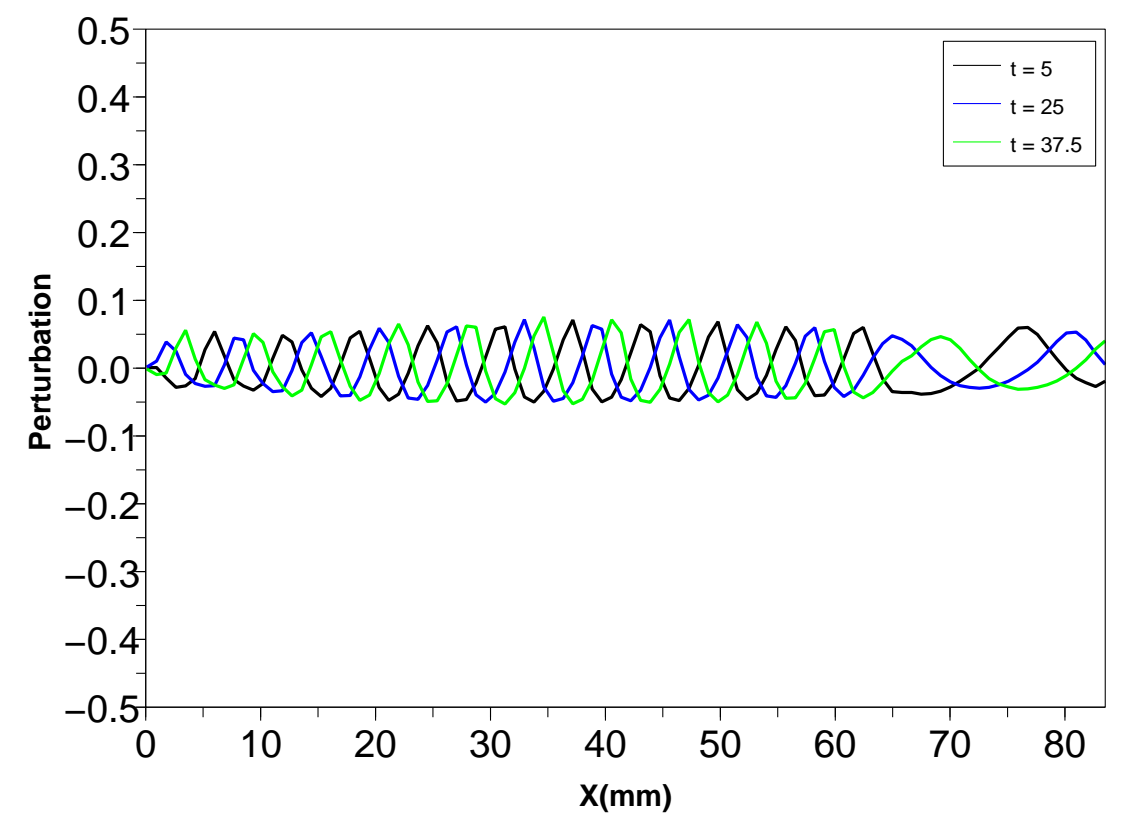

Figure 11: Spatial evolution for various time steps of relative perturbation for a forcing frequency of $1 \mathrm{~Hz}$ in the outlet zone; processing conditions correspond to the unstable experimental case 4 

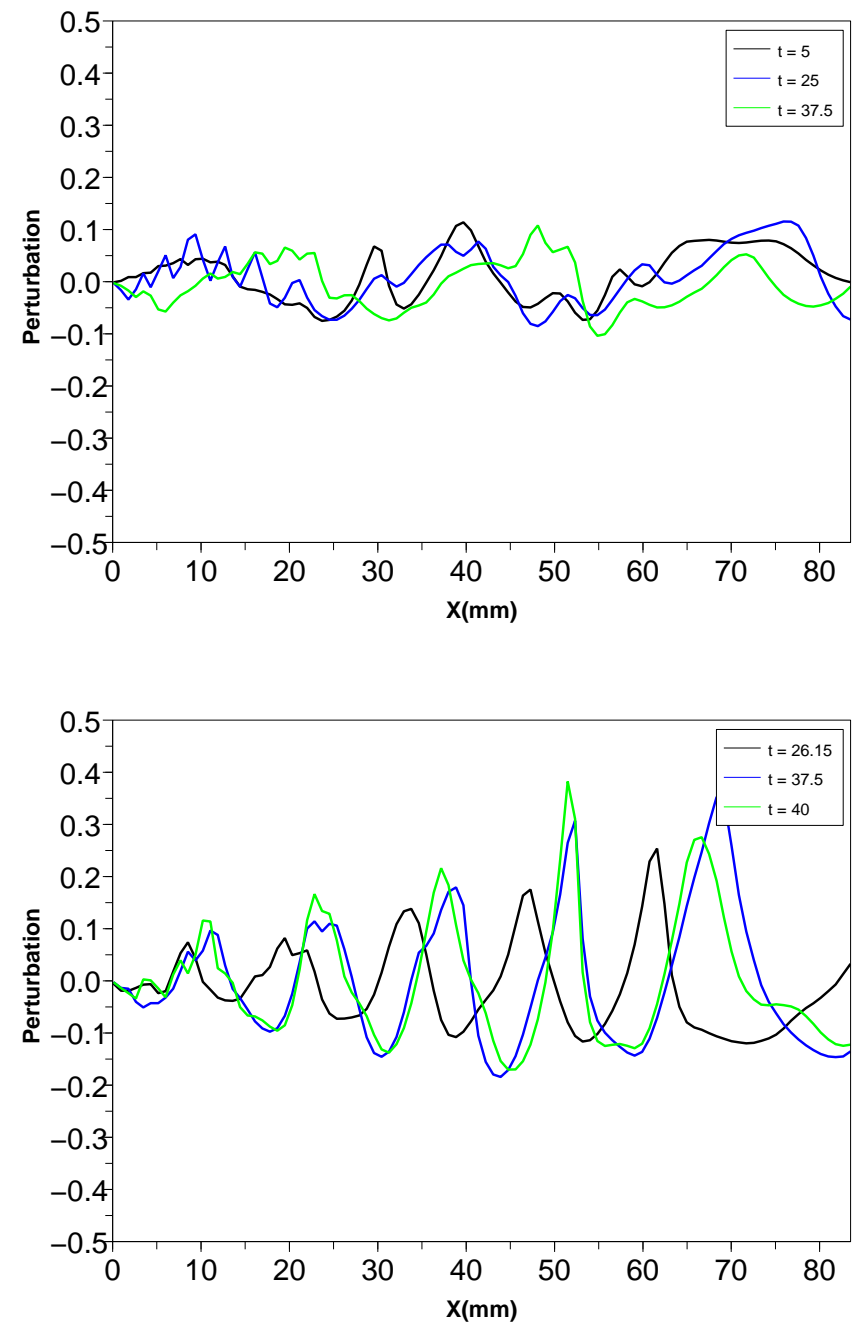

Figure 12: Spatial evolution of the relative perturbations for various time steps for case $\mathrm{N}^{\circ} 4$ and a twice bigger flow rates: a) forcing frequency $0.5 \mathrm{~Hz}, \mathrm{~b}$ ) forcing frequency $1 \mathrm{~Hz}$ 

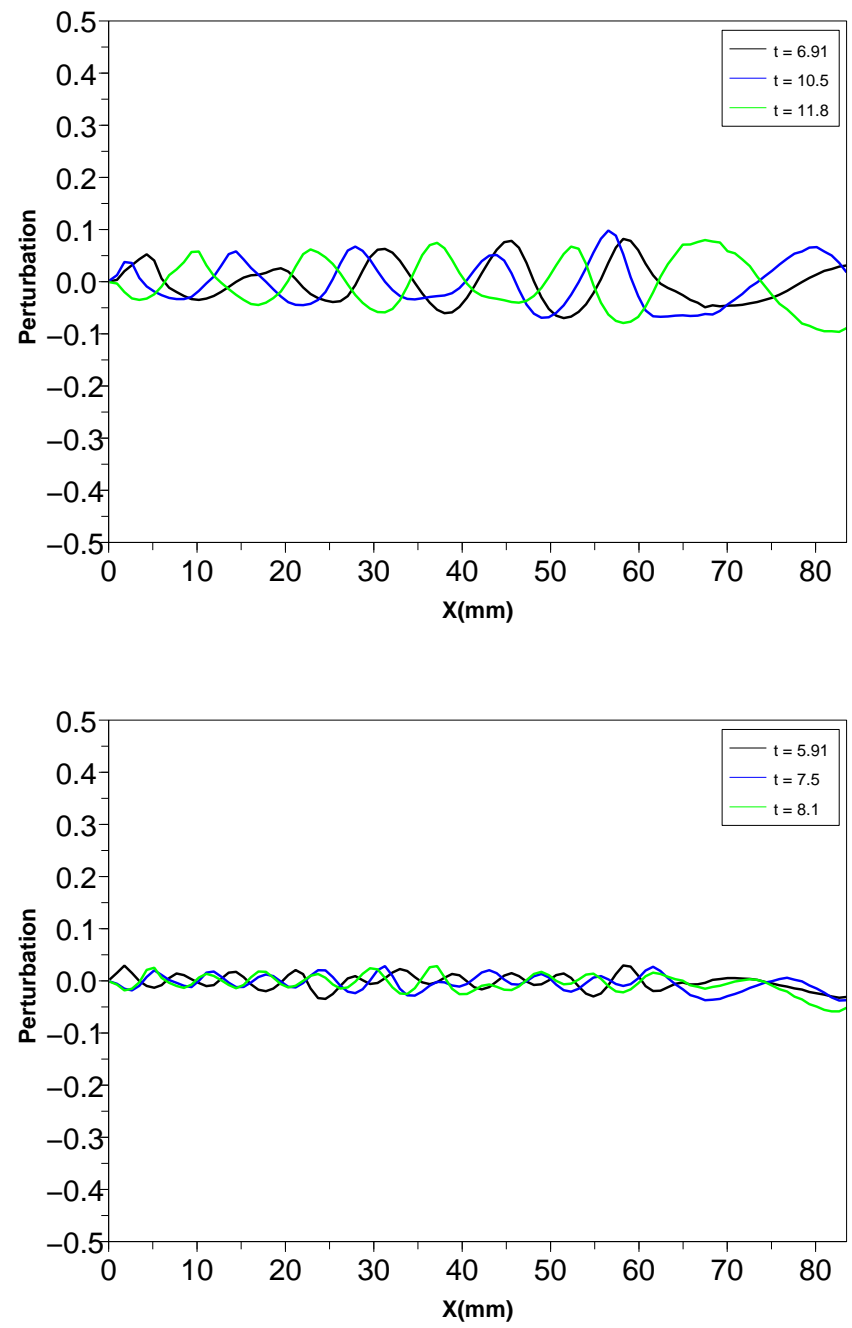

Figure 13: Spatial evolution of the relative perturbations for various time steps for case $\mathrm{N}^{\circ} 4$ and a temperature of $280^{\circ} \mathrm{C}$ : a) forcing frequency $0.5 \mathrm{~Hz}$, b) forcing frequency $1 \mathrm{~Hz}$ 

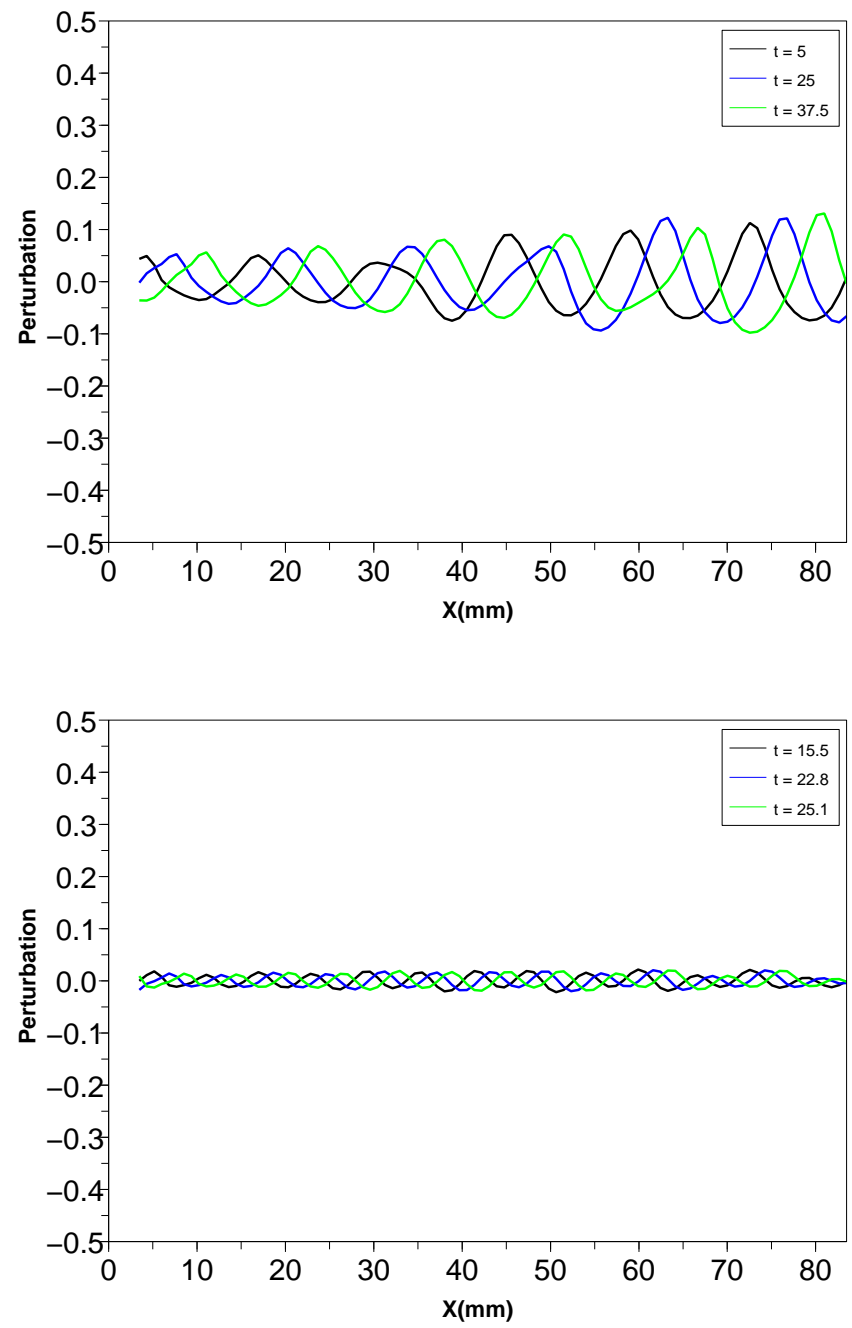

Figure 14: Case $\mathrm{N}^{o} 4$ for a null angle in the convergent geometry. Perturbation along flow motion for various time steps; a) forcing frequency $0.5 \mathrm{~Hz}$, b) forcing frequency $1 \mathrm{~Hz}$ 

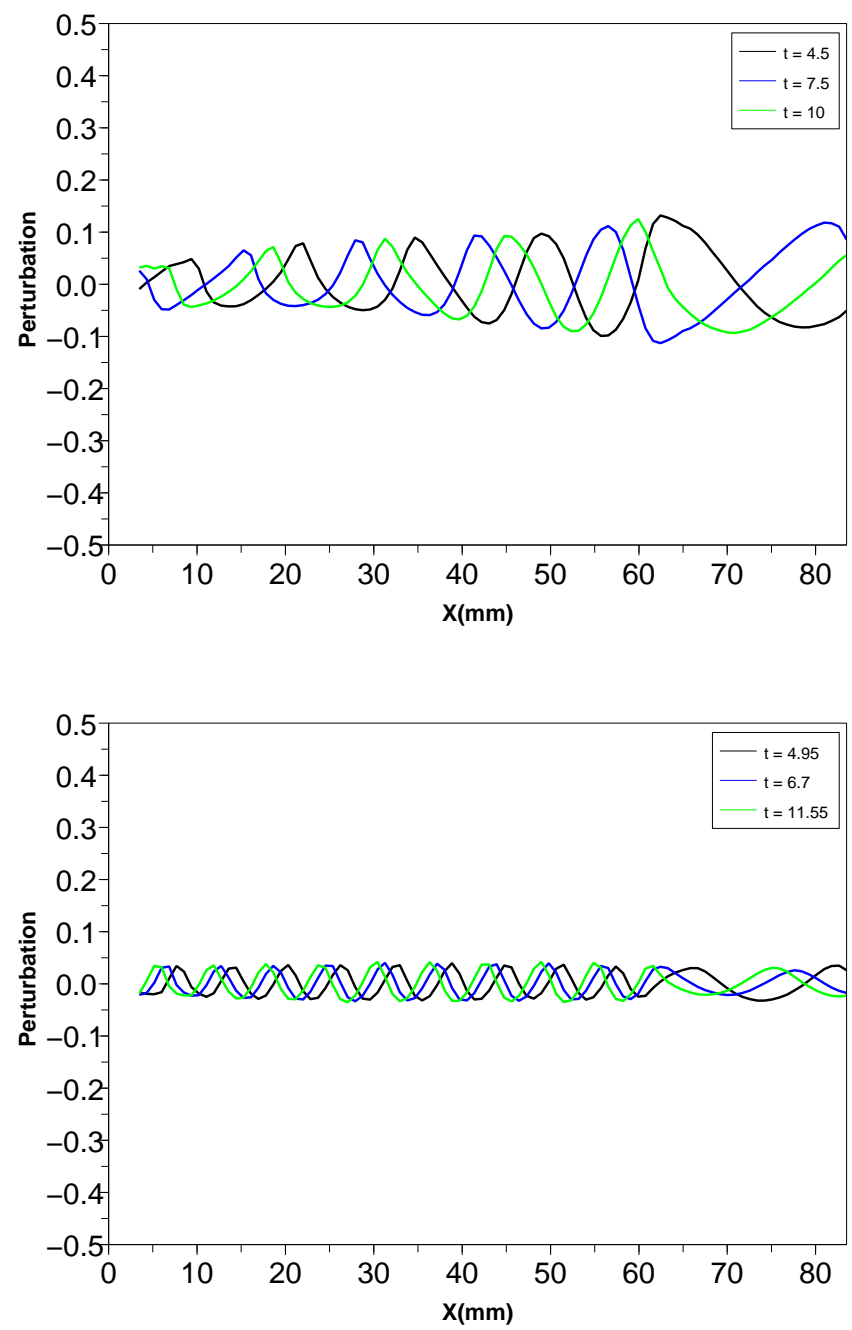

Figure 15: Case $\mathrm{N}^{o} 4$ and $30^{\circ}$ angle in the convergent geometry. Perturbation along flow motion for various time steps; a) forcing frequency $0.5 \mathrm{~Hz}, \mathrm{~b}$ ) forcing frequency $1 \mathrm{~Hz}$ 